\title{
Improved Continuous Positive Airway Pressure Adherence with Pillow Height Change: A Case Report
}

\author{
Hyungee Kim ${ }^{1}$, Jungjoo Lee ${ }^{2}$, Hyeyun Kim ${ }^{3}$ \\ ${ }^{1}$ Medical Student, Catholic Kwandong University College of Medicine, Incheon, \\ ${ }^{2}$ Department of Neurology, Sleep Center, International St. Mary's Hospital, Incheon, \\ ${ }^{3}$ Department of Neurology, Catholic Kwandong University College of Medicine, International St. Mary's Hospital, Incheon, Korea
}

Received April 30, 2019 Revised September 23, 2019 Accepted November 4, 2019

Address for correspondence Hyeyun Kim, MD

Department of Neurology, Catholic Kwandong University College of Medicine,

International St. Mary's Hospital, 25 Simgok-ro 100beon-gil,

Seo-gu, Incheon 22711, Korea

Tel: +82-32-290-3889

Fax: +82-32-290-3879

E-mail: imkhy77@gmail.com
In this case report, we tried to see the relationship between the continuous positive airway pressure (CPAP) adherence of obstructive sleep apnea (OSA) patient and the height of pillow. The patient was 41-year old female with OSA and had trouble adjusting to CPAP therapy due to the high pressure of the CPAP. We performed a overnight polysomnography for 8 hours in hospital and compared the apnea-hypopnea index (AHI) score and optimal CPAP pressure after changing the height of the pillow. The lower pillow setting had lower AHI score and lower optimal CPAP pressure which made the patient to feel more comfortable while sleeping. In conclusion, height of the pillow deserves consideration as a factor to promote CPAP adherence with lowering pressure, especially in the patients who are suffering with high pressure CPAP therapy.

J Sleep Med 2019;16(2):113-115

Key Words: Obstructive sleep apnea, Continuous positive airway pressure, Adherence.
Obstructive sleep apnea (OSA) is a sleep-concerned breathing disorder that involves a decrease or complete stop in airflow in spite of an ongoing effort to breathe. ${ }^{1}$ OSA is an independent risk factor for metabolic disorders such as hypertension and diabetes mellitus, as well as cardiovascular and cerebrovascular disease. For these reasons, it is a disorder that should be properly treated.

Continuous positive airway pressure (CPAP) therapy is a highly effective treatment for the patients with OSA. However, CPAP adherence, how well the patients use it, is a limiting factor to determine the effectiveness on OSA treatment. ${ }^{2}$ There are several ways to increase the CPAP adherence. Lowering level of CPAP pressure is very effective for the patients with severe degree OSA. ${ }^{3}$ This is because, in patients with severe OSA, more pressure is required compared with patients with mild to moderate degree. The high pressure of CPAP is a major cause for patients to suffer from various reasons and to reduce the CPAP adherence rate.

This is an Open Access article distributed under the terms of the Creative Commons Attribution Non-Commercial License (https://creativecommons.org/licenses/by-nc/4.0) which permits unrestricted non-commercial use, distribution, and reproduction in any medium, provided the original work is properly cited.

\section{Case Report}

This patient was a 41-year-old female who had been recognized snoring since several years ago. She had been diagnosed with impaired glucose tolerance and hypertension for the past two years. To manage the metabolic syndrome and obesity, she decided to visit sleep clinic for sleep study and being treated for snoring. Her body mass index was $46.02 \mathrm{~kg} / \mathrm{m}^{2}$. Epworth sleepiness scale was 18.

Overnight polysomnography (PSG) (Nox A1, Nox Medical, Reykjavik, Iceland) was performed for 8 hours in hospital. Her sleep structure showed no sleep stage 3 and rapid eye movement (REM) sleep period and decreased efficiency of sleep (77\%). Apnea hypopnea index (AHI) was 60.5/hr and Supine AHI and non-supine AHI were 75.9 and 59.0/hr, respectively. The minimum oxygen saturation was very low with $48 \%$. Her cephalometric measurement revealed narrow space in superior posterior airway and great distance between the hyoid and the mandible. Superior airway space is narrow $(5.72 \mathrm{~mm})$. The distance between the hyoid and the mandible is significantly increased with $25.03 \mathrm{~mm}$.

In order to have a period of adaptation and training prior to CPAP titration, adaptation period was used as the auto-set- 


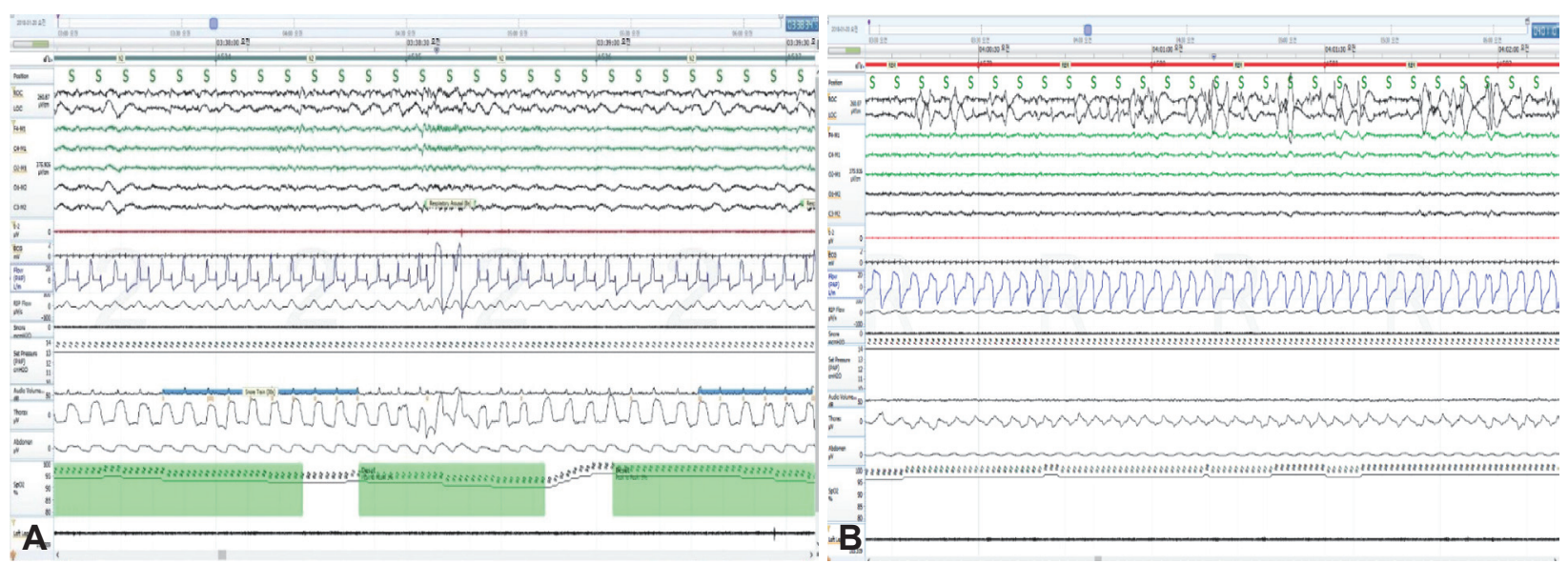

Figure 1. CPAP titration with high-height pillow. (A) CPAP with $13 \mathrm{cmH}_{2} \mathrm{O}$ at stage 2 sleep showed flow limitation. (B) CPAP with $14 \mathrm{~cm}-$ $\mathrm{H}_{2} \mathrm{O}$ at rapid eye movement stage well controlled apnea events. CPAP: continuous positive airway pressure.

Table 1. CPAP titration and adherence summary with pillow height change

\begin{tabular}{cccccccc}
\hline Pillow height & Recording time & CPAP pressure & AHI & $\begin{array}{c}\mathrm{SpO}_{2} \text { saturation } \\
\text { (average) }\end{array}$ & $\begin{array}{c}\mathrm{SpO}_{2} \text { saturation } \\
\text { (lowest) }\end{array}$ & $\begin{array}{c}\mathrm{CPAP} \\
\text { adherence }\end{array}$ & $\begin{array}{c}\mathrm{CPAP} \text { adherence more } \\
\text { than 4 hour per day }\end{array}$ \\
\hline High height pillow & 1.5 hours & $14 \mathrm{cmH}_{2} \mathrm{O}$ & 4.3 & $94.9 \%$ & $90 \%$ & $94 \%$ & $46 \%$ \\
Low height pillow & 2 hours & $13 \mathrm{cmH}_{2} \mathrm{O}$ & 3.9 & $95.2 \%$ & $92 \%$ & $98 \%$ & $87 \%$ \\
\hline
\end{tabular}

CPAP: continuous positive airway pressure, AHI: apnea-hypopnea index

ting CPAP (AirSense 10, ResMed Ltd, Bella Vista, Australia) at home. The average device pressure $\leq 90 \%$ of time for 2 weeks was reported to $14 \mathrm{cmH}_{2} \mathrm{O}$. She struggled with high pressure and complained about the discomfort of air leak forward to her eyes due to the large size of the nasal mask. She had CPAP treatment every night for one month, but the CPAP adherence for more than 4 hour was as low as $46 \%$. We encouraged her to lose weight and regular exercise and she did exercise twice per week and restrict intake hard. However, she failed to weight loss. The average device pressure $\leq 90 \%$ of time have not been lowered. One month later, she underwent manual CPAP titration to make an optimal pressure. In addition, in order to reduce the pressure of CPAP, we proposed to vary the pillow height with CPAP titration. During manual CPAP titration, at the first step, the height of the pillow was $11.5 \mathrm{~cm}$ without lying on the pillow, and the height from floor to the backside of head was $4.5 \mathrm{~cm}$ with lying on the pillow. This pillow was at the same height as her usual pillow. At the first pillow trial during one and half hours, residual AHI was 4.3/hr and pressure of CPAP for eliminating apnea, hypopnea and loud snoring without arousal was 14 $\mathrm{cmH}_{2} \mathrm{O}$ (Fig. 1, Table 1). At the next step, the height of the pillow was $8 \mathrm{~cm}$ without lying on the pillow, and the height from floor to the backside of head was $2 \mathrm{~cm}$ with lying on the pillow. At the second pillow trial, residual AHI was 3.9/hr and the result of CPAP titration during next 2 hour including REM supine period was $13 \mathrm{cmH}_{2} \mathrm{O}$ (Fig. 2, Table 1). The

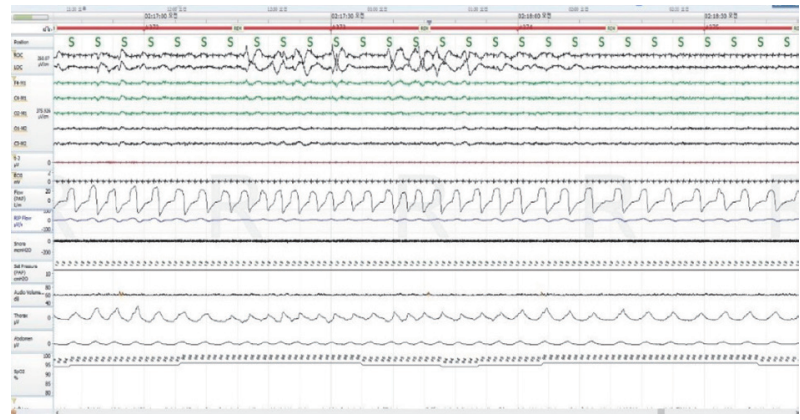

Figure 2. Continuous positive airway pressure titration with lowheight pillow. Optimal pressure with $13 \mathrm{cmH}_{2} \mathrm{O}$ at rapid eye movement/supine stage well controlled the apnea.

pressure of CPAP was reduced by only $1 \mathrm{cmH}_{2} \mathrm{O}$. We prescribed $13 \mathrm{cmH}_{2} \mathrm{O}$ for fixed CPAP treatment with $8 \mathrm{~cm}$ low height pillow. She visited to the outpatient clinic two weeks later, and she said that she was more comfortable using CPAP with new prescribed pressure setting than before. She showed a better adaptation to the use of CPAP for 6 months (Table 1). She has still taken antihypertensive medication, but is better at controlling blood pressure and blood sugar level than before, and regularly exercising as prescribed.

\section{Discussion}

Head position is an important factor to reduce respiratory events in patients with OSA. ${ }^{4}$ In patients with OSA, upper 
airway dimensions at all levels were significantly smaller than the healthy control. ${ }^{4}$ Until now, lateral body position sleep is well known effective treatment option for reducing obstructive events during sleep in some patients with OSA. Recent study showed upper airway collapse is less severe during head rotation than in lateral head and trunk position. ${ }^{5}$ In drug induced sleep endoscopic analysis in OSA, head rotation improved upper airway collapse in supine body position. ${ }^{6}$ Based on several researches, the head position have been a significant factor for aggravating OSA and modifying factor for improving OSA as well as body position, recently. Some researchers have suggested that head position should be included in PSG recording along with body position. ${ }^{7}$

Head position during night sleep is affected by the type and height of the pillow in use. A few studies have reported that pillows used for extension of the cervical spine helped snoring and apnea in patients with OSA. ${ }^{8,9}$ However, the height and shape of the pillow are not clinically suggested for therapeutic purposes.

In severe OSA patients who required higher pressure of CPAP therapy than mild/moderate OSA patients, the effort to reduce the pressure slightly is important that directly affects the CPAP adherence. CPAP adherence was reported to be lower when the CPAP pressure was greater than $12 \mathrm{cmH}_{2} \mathrm{O}$ which make more nasal problems. ${ }^{10}$ Therefore, we should make additional concerns to increased CPAP adherence in patients with CPAP therapy using higher pressure over $12 \mathrm{~cm}$ $\mathrm{H}_{2} \mathrm{O}$. It is necessary to consider the leak during CPAP treatment, the size and type of mask for patients' comfortable use. And the device setting depending the various type of CPAP machine is also considered to increase adherence of CPAP therapy. Until now, head position and pillow effect are not major consideration in CPAP user with severe OSA as well as therapeutic effects on OSA. However, as mentioned above, these factors might play an important role in OSA patients as modifying the posterior airway space and prevent airway collapsing with extension of cervical spine. Based on this case, applying the pillow modification to all CPAP therapy is still week. It is needed to more researches for the effects of the height of pillow and head position for adherence of CPAP therapy.

In conclusion, height of pillow deserves consideration as a factor to promote CPAP adherence with lowering pressure, especially in the patient who are suffering with high pressure

\section{CPAP therapy.}

\section{Acknowledgments}

I want to thank my professor Kim Hyeyun for giving me this opportunity to write a case report and guiding me throughout the study.

\section{Conflicts of Interest}

The authors have no potential conflicts of interest to disclose.

\section{ORCID iDs}

$\begin{array}{ll}\text { Hyungee Kim } & \text { https://orcid.org/0000-0001-5974-4037 } \\ \text { Jungjoo Lee } & \text { https://orcid.org/0000-0002-7414-1936 } \\ \text { Hyeyun Kim } & \text { https://orcid.org/0000-0002-8008-5539 }\end{array}$

\section{Author Contributions}

Conceptualization: Hyeyun Kim. Data curation: Hyungee Kim, Jungjoo Lee, Hyeyun Kim. Formal analysis: Hyungee Kim, Hyeyun Kim. Investigation: Hyeyun Kim. Methology: Hyungee Kim, Hyeyun Kim. Supervision: Hyeyun Kim. Writing—original draft: Hyungee Kim, Hyeyun Kim. Writing-review \& editing: Hyeyun Kim.

\section{REFERENCES}

1. Arnold J, Sunilkumar M, Krishna V, Yoganand SP, Sathish Kumar M, Shanmugapriyan D. Obstructive sleep apnea. J Pharm Bioallied Sci 2017;9(Suppl 1):S26-S28.

2. Baratta F, Pastori D, Bucci T, et al. Long-term prediction of adherence to continuous positive air pressure therapy for the treatment of moderate/severe obstructive sleep apnea syndrome. Sleep Med 2018;43:6670 .

3. Crawford MR, Espie CA, Bartlett DJ, Grunstein RR. Integrating psychology and medicine in CPAP adherence--new concepts? Sleep Med Rev 2014;18:123-139.

4. Tong M, Sakakibara H, Xia X, Suetsugu S. Compensatory head posture changes in patients with obstructive sleep apnea. J Tongji Med Univ 2000;20:66-69.

5. Safiruddin F, Koutsourelakis I, de Vries N. Upper airway collapse during drug induced sleep endoscopy: head rotation in supine position compared with lateral head and trunk position. Eur Arch Otorhinolaryngol 2015;272:485-488.

6. Safiruddin F, Koutsourelakis I, de Vries N. Analysis of the influence of head rotation during drug-induced sleep endoscopy in obstructive sleep apnea. Laryngoscope 2014;124:2195-2199.

7. van Kesteren ER, van Maanen JP, Hilgevoord AA, Laman DM, de Vries N. Quantitative effects of trunk and head position on the apnea hypopnea index in obstructive sleep apnea. Sleep 2011;34:1075-1081.

8. Kushida CA, Rao S, Guilleminault C, et al. Cervical positional effects on snoring and apneas. Sleep Res Online 1999;2:7-10.

9. Makofsky HW. Snoring and obstructive sleep apnea: does head posture play a role? Cranio 1997;15:68-73.

10. Andrade RG, Piccin VS, Nascimento JA, Viana FM, Genta PR, Lorenzi-Filho G. Impact of the type of mask on the effectiveness of and adherence to continuous positive airway pressure treatment for obstructive sleep apnea. J Bras Pneumol 2014;40:658-668. 